\title{
ACOMPAÑAMIENTO TERAPÉUTICO A UN BEBÉ DESPROVISTO DE LAZOS FAMILIARES DESDE SU NACIMIENTO E INSTITUCIONALIZADO
}

Nicole Muñoz Albarrán - Universidad Alberto Hurtado

\begin{abstract}
Nicole Muñoz Albarrán
Psicóloga clínica Miembro fundador del Centro de Estudios y Atención a la Temprana Infancia, Departamento de Postgrado Universidad Alberto Hurtado
\end{abstract}

\section{Correspondencia}

Nicole Muñoz

Dirección: Padre Luis de Valdivia 368, departamento 101, Santiago Centro, Chile.

Teléfono: 935538620

Correo electrónico: nimunoz@uc.cl 


\title{
Resumen
}

En Chile, la mayor parte de los recién nacidos que no son reclamados por sus familias de origen al nacer, son derivados al sistema de protección y sometidos a una institucionalización temprana, careciendo el bebé de una figura de afecto exclusiva y estable. Las condiciones institucionales producen y sostienen muchas veces dinámicas de funcionamiento deshumanizadas, realizando así una oferta vacía de identidad para cada niño/a. El presente trabajo, relata la experiencia de acompañamiento terapéutico a un bebé desprovisto de lazos familiares desde su nacimiento e institucionalizado, abriendo preguntas sobre el trabajo terapéutico a realizar en dichos contextos de sufrimiento.

Palabras clave: psicología infantil; abandono infantil; institucionalización temprana; deprivación; constitución psíquica

\begin{abstract}
In Chile, most of newborns who are not claimed by their families of origin at birth are referred to protection system and are subject of an early institutionalization (Child Care Program). Therefore, the baby lacks of an exclusive and stable affect figure. Institutional conditions often produce and sustain dehumanized functioning dynamics, thus making an empty offer of identity for each child. The present report relates the experience of therapeutic accompaniment to an infant lacking of any family ties since birth and institutionalized, arising questions about the therapeutic work to be done in such contexts of suffering.
\end{abstract}

Keywords: child psychology; child abandonment; early institutionalization; deprivation; psychic constitution 


\section{Introducción}

Las primeras vinculaciones son de suma relevancia para la constitución de la subjetividad, y ésta se encuentra ligada a aquellas personas que han representado para el niño/a sus principales referentes (Lebovici \& Soulé, 1973, Brazelton \& Cramer, 1993). Piera Aulagnier enfatiza que para que la vida somática se preserve es preciso que el medio pueda satisfacer las necesidades del cuerpo real, pero para que la vida psíquica se preserve es igualmente insoslayable la necesidad de la existencia de otro que lo cargue de sentido, manipule su cuerpo real, lo mire, lo toque, lo hable, se anticipe a lo que siente; es insoslayable la necesidad de otro comprometido afectivamente de manera profunda que lo nombre, cualifique e historice (Aulagnier, 1975; Hornstein, 1991). Por el contrario, el cuerpo tomado y manipulado solo en su dimensión biológica, niega la entrega de significaciones estructurantes, llevando a cuestas la sombra del no deseo, de la pulsión de muerte (Hornstein, 1991).

Por lo anterior, la experiencia de aquellos bebés que no son reclamados por sus familias de origen al nacer y son puestos a cargo del sistema asistencial chileno, así como su posterior internación en sistemas residenciales son realidades dramáticas (Jaar, 2013). Para el psicoanálisis el problema de las institucionalizaciones precoces ha tenido un lugar importante en los distintos trabajos de quienes conceptualizaron el trabajo con niños. Autores como Ana Freud, Bolwby, Winnicott y Spitz, contribuyeron a la visualización de los múltiples y nocivos efectos en el desarrollo del infante provocados por la ausencia de figuras significativas en el origen (Arteaga \& Martínez, 2017). René Spitz (1969), en sus pioneras observaciones de niños/as institucionalizados documentó el crudo y doloroso impacto de la privación afectiva. En circunstancias de abandono total de su familia de origen, y cuando los cuidados son administrados en forma anónima, sin que pueda establecer un lazo afectivo significativo con alguno de sus cuidadores, se establece un 
conjunto de perturbaciones somáticas y psíquicas que este autor agrupó bajo el nombre de hospitalismo.

A pesar que el sistema de protección para niños vulnerados en sus derechos ha adquirido particular connotación en los últimos años en Chile (Muñoz, Fischer \& Chía, 2013), para autores como Matías Marchant (2014) las condiciones institucionales chilenas continuarían produciendo y sosteniendo dinámicas de funcionamiento deshumanizadas, donde el afecto personalizado de alguien que acoja su dolor y done sus esperanzas, simplemente está ausente; obligando a todos sus miembros a una experiencia homogénea, violentando toda posibilidad de diferencia, realizando así una oferta vacía de identidad para cada niño y niña. Así, "si muchos de nosotros pensábamos que el hospitalismo era un concepto histórico y que habían cambiado las condiciones de cuidado de estos menores en los servicios asistenciales de lactantes, (estos) hallazgo(s) constituye(n) una cruda rectificación de dicha apreciación” (Jaar, 2013).

En la revisión realizada no se encontraron en el país alternativas de tratamiento terapéutico que consideren la atención del aislamiento afectivo de los bebés institucionalizados, enfocados particularmente en brindar una relación de afecto estable, exclusiva y afectiva desde la cual puedan sentirse sostenidos. En este marco desde el año 2014 la Corporación de Estudios y Atención a la Temprana Infancia ha implementado un método de abordaje psicológico, intensivo y estable, que posee la finalidad de mitigar y prevenir el cuadro carencial de algunos de estos/as niños/as, en el que un profesional de la salud mental se orienta a la construcción junto al niño/a de una relación de afecto exclusiva y recíproca (Jaar, 2013). Este modelo de intervención es una aplicación del método de observación de Esther Bick (1964), siguiendo el esquema de los tres tiempos de realización propuestos por la autora: la sesión diaria con el bebé, la redacción de los sucesos y respuestas emocionales en las 24 horas sucesivas y la participación en un grupo de trabajo donde 
relata su experiencia semanal con el bebé y donde es sostenido por el monitor y los integrantes del equipo. Asimismo, considera los aportes realizados en el abordaje terapéutico de Francoise Dolto (1984) y Carolina Eliachef (1994), pioneras en la utilización de intervenciones verbales francas y directas con los/as niños/as para otorgar sentido a sus manifestaciones sintomáticas (Jaar, 2013).

\section{Acompañamiento terapéutico de un bebé desgarrado de sus primerísimos vínculos e}

\section{institucionalizado}

Como miembro de la Corporación de Estudios y Atención a la Temprana Infancia, inicié el acompañamiento de Alejandro desde el $18^{\circ}$ día de su vida y tengo sesiones de $1-1$ 1/2 hora, los siete días de la semana. Su madre había abandonado el hospital "sin querer mirarlo". Se trataba de una mujer joven, adicta a la pasta base de cocaína. Su nombre, por tanto, había sido dado por el personal técnico del hospital.

Cabe señalar, que los primeros dos meses de vida Alejandro permaneció en la unidad de cuidados mínimos de un hospital de la Región Metropolitana de Chile, para posteriormente ser derivado a una casa de acogida de lactantes dependiente del Servicio Nacional de Menores del país.

Alejandro es un niño hermoso, pequeño, de ceño fruncido, fue para mí, un amor a primera vista. Asumí la tarea de acompañarlo y cuidarlo buscando principalmente crear una relación afectiva. Para ello, mis principales herramientas fueron mi presencia regular, estable y afectuosa; y el tenerlo en mi mente, constantemente, aun cuando su situación de vida me generaba dolor y ansiedad.

A los pocos días de iniciada esta experiencia, Alejandro ocupó mi tiempo, energía y dedicación. Sus rutinas se volvieron las mías, buscando posicionarme como una figura que se adaptara a sus necesidades y que se constituyera por tanto como un espacio distinto al que se le 
entregaba, donde era él quien debía acomodarse a horarios y formas ordenadas desde las instituciones. Parte importante de mi rol era estar allí en los momentos de su alimentación, muda y baño. Tocarlo, estimularlo, acunarlo, mimarlo, pensarlo, hablarlo; anudarlo a este lazo rápidamente establecido, se volvió el motor de mi quehacer. Lo nombraba por su nombre, le hablaba de su historia, de su soledad, le hablé de mí y le expresaba mi alegría de verlo y acompañarlo. En las primeras semanas en el hospital, me impresionaba como un bebé apenas vivo. Apenas lloraba, se despertaba o movía. Para el personal de la institución esto correspondía a un “se porta súper bien”, mientras que a mí me atemorizaba su muerte.

El día que fue trasladado desde el hospital a la Casa de Acogida, lo vi cerrar sus ojos y puños estando despierto, sin reaccionar a ningún estímulo. Su temperatura corporal subió y creí ver sus esfuerzos por autocontenerse. Lo vi posteriormente mostrar aversión a mi mirada por largos periodos durante mis visitas, ¿resintiendo nuestra dolorosa separación diaria, sus necesidades de vínculo y afecto día a día frustradas? Lo vi ser objeto de indicaciones arbitrarias propias de las reglas de una institución de menores que debe mantener un orden y rutinas: obligado a comer, a dormir, a quedarse en su cuna. Alejandro no tiene juguetes ni ropa propia: "Esos calcetines un día los ocupa uno, se lavan y los ocupa otro y así’.

Quisiera también mencionar algunos hitos de la vida de Alejandro y de nuestro vínculo, que se inscribieron como momentos de encuentro: Él partió focalizando su mirada en mí mientras tomaba su leche. Alrededor del mes y medio me seguía con la mirada, exploraba mi rostro, y jugó con mi cabello mientras tomaba su leche desde los tres meses hasta el final de nuestros encuentros. Alrededor de los dos meses también reconocía mi llegada. Primero en el hospital, se mantenía despierto a la hora en que regularmente lo visitaba, y hacia los tres meses crecientemente fue sonriéndome, agitando sus manos y piernas, hasta mostrarme una evidente alegría. Reconocía mi 
voz a la distancia tras mi llegada, a los tres meses de manera creciente, si estaba llorando; dejaba de hacerlo al escucharme, o bien comenzaba a llorar dirigiendo su mirada hacia mí para ser alzado en mis brazos. A los tres meses y 10 días, respondió a mi “agú” con su “agú” y ensayamos diálogos hasta su partida del hogar. Hacia sus cuatro meses, siendo el único bebé de su sala que contaba con una figura de acompañamiento estable, aprendió a extender sus brazos para ser alzado ¿Él se sentía reconocido? Algo de eso se fue instalando en Alejandro.

Comenzó así a explorar su entorno, su salud era fuerte y fue logrando un desarrollo psicomotriz casi normal para sus meses de desarrollo, aspectos que lo diferenciaban dolorosamente del resto de niños/as de su sala. Así también, logramos separarnos con creciente tranquilidad. Alejandro fue quien creciendo y progresando en su desarrollo me fue entregando también seguridad para apartarnos diariamente. Así también comencé yo a sentir que podíamos separarnos a diario confiando en reencontrarnos. Y así nos hallamos durante 249 días, hasta el encuentro con su familia adoptiva.

\section{Notas finales}

Considerando que no existen suficientes registros de investigaciones que pongan acento en las intervenciones y en otras acciones que consideren la realidad de bebés desprovistos de lazos familiares, parece crucial obtener una mayor experiencia en la aplicación de esta metodología para evaluar sus aportes en el desarrollo del psiquismo de estos infantes; pudiendo desde este quehacer contribuir con evidencia científica al conocimiento de la infancia temprana vulnerada, a la responsabilidad social de abordar y visibilizar la dolorosa vivencia de estos niños y niñas.

Sin perjuicio de lo anterior, el poner en palabras la experiencia de Alejandro y la de acompañarlo, pretende no circunscribir la realidad tan solo de la institucionalización temprana, si no abrir preguntas sobre las intervenciones que tienen lugar en la infancia, así desde su cercanía y 
cotidianeidad cuestionar lo que Aulagnier propone como preguntas que tengan sentido ante la propia escucha.

Mi meta profesional con Alejandro fue cuidarlo con la añoranza de establecer un vínculo de significación emocional recíproco, que él pudiese así experimentar la presencia de otro mirándolo, hablándolo, ordenando su historia; pero también tocándolo, acunándolo, acariciándolo; mi meta fue que él pudiese experimentar la presencia de otro amándolo. Quisiera aquí detenerme para hacerme cargo de cuestionamientos que a lo largo de esta experiencia se me realizaron, que me interpelaron y me llevaron a enfrentar mis propias concepciones sobre el quehacer clínico: ¿Qué hay de psicoanálisis en esta intervención que erige como centro del trabajo el establecimiento de una relación de afecto profundo y que toma el cuerpo del "paciente” ?, o más allá ¿Qué hay de terapéutico en esta intervención?

Los modelos tradicionales de abordaje psicoterapéutico de infantes por lo general ponen énfasis en las intervenciones verbales aclaratorias y de carácter mutativas propias de la teoría psicoanalítica. Así, es posible encontrar entre otros autores a Caroline Eliachef (1994), quien plantea que simbolizar el sufrimiento de niños institucionalizados debe orientarse a intervenir, sin tocarle.

Desde la vivencia de acompañamiento terapéutico a Alejandro, es posible dar cuenta que el contexto chileno interpela a las intervenciones psicoanalíticas a pensarse en un campo distinto y poco explorado, donde no solo es requerido reparar y realizar interpretaciones; sino a realizar intervenciones estructurantes (Janin, 2012), abriéndose a nuevas preguntas: ¿cómo separar el rol de terapeuta o de maternaje en dichos contextos de sufrimiento? y fundamentalmente ¿es necesario hacerlo? 


\section{Referencias}

Arteaga, M. \& Martínez, M. (2017). Breve historia conceptual y técnica que sustenta la teoría y la clínica de los modelos de intervenciones terapéuticas en vínculos tempranos. En J. Navarro, F. Pérez \& M. Arteaga (eds.), Vínculos Tempranos, Transformaciones al inicio de la vida (pp. 91-118). Santiago de Chile: Ediciones Universidad Alberto Hurtado.

Aulagnier, P. (1975). La violencia de la interpretación. Buenos Aires: Amorrortu.

Bick, E. (1964). Notas sobre la observación de lactantes en la enseñanza del psicoanálisis. The International Journal of Psycho-Analysis, XVL, 4.

Brazelton, T. B. \& Cramer, B. (1993). La relación más temprana: padres, bebés y el drama del apego inicial. Barcelona: Paidós Ibérica.

Dolto, F. (1984). Seminario de psicoanálisis de niños 1. Siglo veintiuno editores.

Eliachef, C. (1994). El cuerpo y la palabra: Ser psicoanalistas con los más pequeños. Buenos Aires: Nueva Visión.

Hornstein, L. (1991). Cuerpo, historia, interpretación. Piera Aulagnier, de lo originario al proyecto identificatorio. Buenos Aires: Paidós.

Jaar, E. (2013, mayo). Un modelo de intervención en la privación afectiva temprana. Trabajo presentado en VII Congreso de la Federación Latinoamericana de Psicoterapia Psicoanalítica y Psicoanálisis (FLAPPSIP). Santiago de Chile, Chile.

Janin, B. (2012). Las intervenciones del psicoanalista en psicoanálisis con niños. Cuadernos de Psiquiatría y Psicoterapia del niño y del adolescente, 49-56.

Lebovici, S. \& Soulé, M. (1973). El conocimiento del niño a través del psicoanálisis. México: Fondo de Cultura Económica. 
Marchant, M. (2014, 24 de abril). Y después de los horrores en el Sename, ¿qué? Ciper. Recuperado el 12/11/2017, de http://ciperchile.cl/2014/04/24/y-despues-de-los-horroresen-el-sename-\%C2\%BFque/

Muñoz, C., Fisher, C. \& Chía, E. (2013). Lineamientos estratégicos para modelos de cuidado alternativo dirigidos a niños/as menores de seis años bajo protección estatal. En Centro de Políticas Públicas UC (2013) Propuestas para Chile (pp. 115-150). Recuperado el 11/05/2017 de http://politicaspublicas.uc.cl/publicacion/concurso-de-politicas-publicas2/propuestas-para-chile-2013

Spitz, R. (1969). El primer año de vida del niño. Buenos Aires: Fondo de Cultura Económica.

Recepción: 28 de mayo de 2018

Última Revisión: 23 de noviembre de 2018

Aceptación Final: 25 de noviembre de 2019 